\title{
An integrated digital microfluidic bioreactor for fully automatic screening of microalgal growth and stress-induced lipid accumulation
}

\author{
Yunhua Wang ${ }^{1}$, Hongyu Zhao ${ }^{1}$, Xianming Liu $^{2}$, Lin Wang ${ }^{1}$, Youwei Jiang ${ }^{3}$, Jianfeng Li $^{4}$, \\ and Guoxia Zheng ${ }^{1}$ \\ ${ }^{1}$ Dalian University \\ ${ }^{2}$ Dalian Institute of Chemical Physics \\ ${ }^{3}$ Southern University of Science and Technology \\ ${ }^{4}$ Jiangsu Celyee Cell Technology Research Institute
}

July 29, 2020

\begin{abstract}
Algae are promising feedstock of biofuel. The screening of competent species and proper fertilizer supply are of the most important tasks. To accelerate this rather slow and laborious step, we developed an integrated high-throughput digital microfluidic (DMF) system that uses discrete droplet to serve as micro-bioreactor, encapsulating microalgal cells. Based on the fundamental understanding of various droplet hydrodynamics induced by the existence of different sorts of ions and biological species, an incorporation of capacitance-based position estimator, electrode-saving-based compensation and deterministic splitting approach was performed to optimize the DMF bioreactor. Thus, it enables all processes (e.g. nutrient gradient generation, algae culturing and analyzing of growth and lipid accumulation) occurring automatically on-chip especially in a high-fidelity way. The ability of the system to compare different micro algal strains on chip was investigated. Also, the Chlorella sp. were stressed by various conditions and then growth and oil accumulation were analyzed and compared, which demonstrated its potential as a powerful tool to investigate microalgal lipid accumulation at significantly lower laborites and reduced time.
\end{abstract}

\section{Introduction}

Microalgae are of particular interest as the most promising source for sustainable energy which has the potential to meet the increasing global demand for transport fuels by displacing non-renewable fuels. Although biodiesel production extracted from microalgae is proved to be technically practicable, a scalable and commercially viable are far from competitive fossil fuels' prices (Jin et al., 2020). Thus, new methods must be developed to increase yield and decrease the cost of the production. Currently, the improvements aiming at algal lipid accumulation mainly include screening suitable microalgal species which grows fast and/or could produce more oil, and optimizing their growth conditions (e.g., nutrients, temperature, $\mathrm{pH}$ and light condition, etc.), as well as under-expressing genes involved in lipid biosynthesis pathways (Chen and Chang, 2016; Khandelwal et al., 2020; Richter et al., 2018). All screenings are typically done by classical laboratory culturing and off-line lipid analyzing methods. In many cases, these processes are costly and labor-intensive. It is especially true in cultivation conditions optimizing experiments. Large scale bioreactors and repetitive dispensing induced by parallel experiments and concentration gradients generation make it extremely physical space exhausting and labor-intensive. Thus microalgal lipid screening could benefit from a more miniaturized, streamlined and integrated approach. The maneuverable electrowetting-on-dielectric (EWOD) behavior of micro-droplets, on variable-electrized surface, has impelled the development of digital microfluidic (DMF) systems for high-throughput micro-reactors (Gao et al., 2013), which have prop up a 
wide range of biological and chemical applications in sub-microliter/or nanoliter drop scales, such as cellbased screenings (Han et al., 2019), immunoassays (Rackus et al., 2015), polymerase chain reactions (Schell et al., 2012), and single crystal synthesis and crystallization (Josep et al., 2014). DMF achieves a droplet manipulation via surface-tension modulation induced by electrodes coated with dielectric and hydrophobic materials. It belongs to a two-dimensional planar electronic control system (Fan et al., 2011), in which complex experimental procedures (eg. concentration gradient generation, cell staining and analyzing, etc.) can be effectively carried out through basic fluidic operations including droplet dispensing, moving, splitting, mixing and merging (Wang et al., 2017). Their open structure (i.e., no preset microchannel), scalability and high compatibility with diverse aqueous and different operational protocols, thus offering a more flexibly controllable platform for lab-on-a-chip applications (Choi et al., 2012).

Despite these tremendous potentials, only a few labs have reported application of DMFs-based platforms in microalgae screening at present. Reliability and robustness are still great challenges of DMF based microbioreactors due to the co-existence of different sorts of chemicals and biological species. Our experiments data substantiated that droplets containing various nutrient ions, salinity and algae species ran at dissimilar velocities and so the optimal charging times. Especially, low-moving algae droplets usually could not reach the target electrodes due to insufficient charging time, halting the entire system. To overcome potential driven failures, a feedback control loop, especially independent from droplet constituents should be included into DMF control system. All droplets were highly desirable to be addressed by real- time position-dependent maneuver mode to avoid manual tuning, and a self-directed expert control to guide multi-droplet manipulation. Furthermore, for stressor-induced lipid assays, which involve many steps of component dilution, the consistency of dispensed droplet volumes is also pivotal. However, currently, in most DMF applications highly sensitive to sample volume variations (e.g., immunoassays (Vergauwe et al., 2011), DNA amplification (Chang et al., 2006; Malic et al., 2009) and cell analyzing (Bogojevic et al., 2012), droplet generation approaches showed a $\sim 10 \%$ variability. These applications leave the cutting and generating process subject to many uncertainties, and thus affect reproducibility.

Herein, we demonstrated an improved DMF-based algae micro-bioreactor enabling a comprehensive implement of culturing and stressors triggering lipid accumulation analyses in microalgae, especially a highfidelity screening performance of fertilization applied in brackish/salt water, which will make microalgaebased biofuels more attractive and competitive. Three optimizations were involved in the development of the DMF-bioreactor. The first one is that the imperfect profiling of droplet hydrodynamics are inspired by the capacitance-sensing concept. In this work, the droplet-derived capacitance was determined in real time and was used to track position (Zhang et al., 2019). Thus, droplets can be automatically addressed in real time, without human observation, pre-calibration (like in voltage feedback, Shih et al., 2011 ), or image capturing/processing (like in visual feedback loop, Shin and Lee, 2010). The second optimization is electrode-saving-based compensation. More electrodes of the DMF will lead to increasing of complexity of control system and feedback circuit. To compensate the system complexity and cost-adding, an electrodesaving scheme with inherent simplicity and fault tolerance was applied here. In this scheme, electrodes for algae culturing are connected with "finger-fringe" geometry rather than one or more interval electrodes. The third improvement of the developed bioreactor is deterministic splitting of liquid. A simple approach called "bi-directional stretching spiting was used to achieve accurate splitting of liquids. It can significantly reduce the splitting volume variation and avoid abnormal droplet dividing, which is usually occurred in traditional unidirectional stretching-split process.

We evaluated the DMF prosperities by measuring actuation fidelity, volumetric variation in splitting and fluorescence intensities of target droplets, as well as DMF compatibility in algae. The ability of the system to compare different microalgal strains on chip was investigated. Also, the selected Chlorella sp. was successfully stressed by various fertilizer conditions and then growth and oil accumulation were well analyzed and compared. Thus the presented system allows, for the first time, the entire proceed in of quantifying microalgal proliferation and stressor-induced lipid enrichment were performed on a microchip, smoothing the way towards more reliable and automated algae lipid screening. 


\section{Materials and Methods}

\subsection{DMF screening system}

The developed DMF screening system consists of three subsystems: (a) the chip, (b) the operation unit, and (c) the detector element (Fig. 1). The chip is composed of two parallel plates and integrated with a temperature controller. The photograph of 24 electrodes chip demonstration was shown in Fig.1a. In 24 electrodes, there are 3reservoir electrodes(for nutrients sample, blank and algae/probe, respectively, with the size of $2 \times 2 \mathrm{~mm}$ ), 1 waste electrode for discarding droplet (with the size of $4 \times 4 \mathrm{~mm}$ ), 2 preparing electrodes for droplet waiting (with the size of $2 \times 2 \mathrm{~mm}$ ), 6 culturing electrodes for addressing algae droplets (with the main size of $2 \times 2 \mathrm{~mm}$ and "finger-fringe" geometry of $2 \mathrm{~mm}$ length) and 12 actuation electrodes to implement basic fluidic operations (moving, splitting, mixing and merging, with the size of $2 \times 2 \mathrm{~mm}$ ). To increase the culturing throughput of a single chip, we applied a simple scheme that only a culturing electrodes array was added onchip and other electrodes were the same as 24 electrodes demonstration. A $6 \times 3$ culturing electrodes array chip were shown in Fig. 1d and the culturing electrodes were connected with "finger-fringe" bridges in each line. To further saving electrodes, $6 \times n$ culturing electrodes were connected with lengthwise "finger-fringe" bridges (Fig. 1e). The materials and fabrication procedure of chip were described in details in Fig. S1 and supporting information. The operation unit is FPGA (field programmable gate array) based actuation system, which is responsible for applying voltage to the electrodes, with a real-time capacitance-based position feedback circuit to insure the accuracy of actuation (Fig. 1b). The Hardware of DMF operation unit and operating principle of the capacitance-based position feedback circuit as well as the entire performance of the DMF micro-bioreactor were described in details in Fig. S2 and S3 and supporting information..

\subsection{Microalgae strains}

Four dominant sea species of microalgae in Chinese coastal waters including Dicrateria zhanjiangensis Hu. Var. sp., Chlorrela. Sp., Platymonas helgolandica var. tsingtaoensis and Platymonas subcordiformis were used in our experiments. Their main characteristics were shown in Table S1 and supporting information.

\subsection{Droplet actuation complexity and reliability}

To assess EWOD actuation complexity and fidelity, a droplet was programmed to move to the adjacent electrode repeatedly for one hundred times. In schema without feedback, actuations were performed using different charging time $\left(T_{a}=100,200,300,400,500,600,700,800,900 \mathrm{~ms}\right)$ for ion samples and $\left(T_{a}=300\right.$, $600,900,1200,1500,1800,2100 \mathrm{~ms})$ for algae samples. In feedback mode, an initial charging time $T_{a}$ of 300 ms was used, with an error-sensing step between two charging durations of $T_{c}=100 \mathrm{~ms}$. A "\% completion" parameter was set as the number of successful droplet movements divided by the total number of movements.

\subsection{Dilution properties of DMF}

$$
E_{n}=\sqrt{n \times E^{2}}, E=\frac{\sum_{i=1}^{m}\left|\frac{\left(S_{i}-1 / 2 S_{0}\right)}{1 / 2 S_{0}}\right| \times 100 \%}{m}
$$

Where, four merge-split steps were the ultimate of our dilution to meet the demand of minimum concentration generation. Therefore, when $n=4, E_{n}$ will be the maximum total error of the diluter. $\mathrm{E}$ is the average value of split volumetric error. $S_{i}$ is the area of a divided droplet of $i$ step.

\subsection{Characterizing of On-chip BODIPY staining}

A stock solution of BODIPY 493/503 (Inc. Eugene, OR, USA) prepared at $10 \mu \mathrm{g} / \mathrm{ml}$ in dimethyl-sulfoxide (DMSO) was use as a fluorescent probe for algae lipid staining. This probe was used for their high selectivity to the hydrophobic tri-acyl-glycerolsaccumulatedin stressedalgal cells (Gocze et al., 1994; Hu et al., 2008). The capability of in-situ lipid analysis in DMF system was characterized in details in Fig S4 and SI text.

\subsection{DMF growth and stressing experiments}


To demonstrate the streamlined analyzing function of the developed system, growth and oil accumulation analyses in algae were performed on-chip. Four marine species of D. zhanjiangenis , Chlorella sp. , P.helgolandica and P.subcordiformis were candidates for algal strains screening. 5-day culture (N-replete culture with $\mathrm{f} / 2$ medium under a light intensity of $100 \mu \mathrm{mol}$ photons $\cdot \mathrm{m}^{-2 *} \mathrm{~s}^{-1}$ with a 12 hour light-dark cycle) with the initial cell density of $5 \times 10^{4}$ cells $/ \mathrm{ml}$ were implemented for growth profile acquiring, and were followed by on-chip oil staining/quantification. The algae growth were characterized using apparent biomass increase rate, respectively:

$V=\frac{\mathrm{LnN}_{t}-\mathrm{LnN}_{0}}{T_{t}-T_{0}}$

Where $V$ represents the apparent biomass increase rate $\left(10^{5}\right.$ cells $\left.\mathrm{ml}^{-1} \mathrm{~d}^{-1}\right), \mathrm{N}_{0}$ and $\mathrm{Nt}$ are the cell density at the beginning and at the end, respectively $\left(10^{5}\right.$ cells ml-1 $)$ and $\left(\mathrm{T}_{\mathrm{t}}-\mathrm{T}_{0}\right)$ during the test $(\mathrm{d})$. Data are showed as mean +- SD. The lipids were analyzed in the same way as described above. These results were compared with off-chip experiments, in which algal were cultured in 96-well plate and analyzed by total lipid extraction (Bligh et al., 1959; Gim et al., 2016). The following experiments were to evaluate the suitability of the DMF system for stressor trigging and in-situ analyzing algal oil accumulation. 5day-DMF culturing of selected (oil-rich/high growth rate) strain (Chlorella. sp. ) was stressed in concentration gradient (CG) droplets and then BODIPY fluorescence responses were observed to screen the optimal fertilizations. The optimized fertilizer conditions include nitrogen-depleted conditions (in the form of nitrate, $\mathrm{NaNO}_{3}$ ) and phosphorusdepleted conditions (in the form of dihydrogen phosphate, $\mathrm{NaH}_{2} \mathrm{PO}_{4}$ ). Three replicates were tested on one chip. All Data are presented as mean +- SD.

\section{Results and discussion}

\subsection{Actuation complexity and fidelity in DMF algae screening system}

DMF device shown in Fig.1 was designed to generate arrays of droplets containing algae, which were exposed to different fertilized salt waters, and interrogated with microalgal growth as well as lipid production measurements. For implement of a high-fidelity EWOD controlled algae micro-bioreactor, we firstly evaluated the droplet actuation reliability without feedback control. As demonstrated in Fig. 2, the displacements of droplets containing multiple liquid evolved in DMF-bioreactor versus the charging time presents a nonlinear relationship. The alterations of the position of the droplets with nutrients during this state are distinct from each other even though with the same equivalent concentration (Fig.2a).The moving velocities for droplets containing DI water, $0.1 \mathrm{moll}^{-1} \mathrm{NaNO}_{3}$ and $0.1 \mathrm{~mol} \mathrm{l}^{-1} \mathrm{NaH}_{2} \mathrm{PO}_{4}$ were $4.99 \mathrm{~mm} \mathrm{~s}^{-1}, 6.52 \mathrm{~mm} \mathrm{~s}^{-1}$ and, 3.51 $\mathrm{mm} \mathrm{s}^{-1}$, respectively. Also, as shown in Fig.2b, the transport distance of salty droplets are different during the actuation time. The movements of sea water with salinity of 10.9 (mainly $\mathrm{NaCl}$ ) which normally exist in brackish/salt water environments (i.e. brackish lake/estuary, costal area and high salt lagoon/open sea), were observed with velocities of $5.12 \mathrm{~mm} \mathrm{~s}^{-1}, 6.47 \mathrm{~mm} \mathrm{~s}^{-1}$ and $6.62 \mathrm{mms}^{-1}$, respectively (Fig.2b). All these profiles of electrolytes, which are usually applied in algae culture condition screening, revealed that the differences in the ions sorts/contents of the liquid droplets also lead to different optimum charging times. Interestingly, the observed velocity-trend is a function of ion kosmotropicity, which represents the capability of an ion to strengthen the water structure (Hribar et al., 2002; Marcus, 1994, 2015). The movements of droplets containing algae suspension are shown in Fig. 2c, with velocities of $2.15 \mathrm{~mm} \mathrm{~s}^{-1}$ for Chlorella. sp. $(C$ cell $)$ droplets and $1.38 \mathrm{~mm} \mathrm{~s}^{-1}$ for P.helgolandica $(P$ cell $)$ droplets. Regarding the slow movement of algae suspension (vs. DI water of $4.99 \mathrm{~mm} \mathrm{~s}^{-1}$ ), it was found that procrastination occurred more seriously in droplets containing $P$ cells than those containing $C$ cells even though with the same cell densities. However, when the mass of the content was taken into account, the mass of $P$ cells in solution will be much higher than that of $C$ cells due to their larger cell size. It has been reported that microalgae (usually $5-100 \mu \mathrm{m}$ in diameter) can be defined as a suspension of organic particles in a fluid. The concentration, size and shape of the microalgae will influence the fluid rheological behavior (Dalmazzo et al., 2010). Particle concentration is main parameter influencing the fluid viscosity. In particular, an increase of particle concentration determines an increase of fluid non-Newtonian behavior instead of the Newtonian one, which is typical for water and solutions with low particle concentration (Mueller et al., 2010). More frustratingly, these non-Newtonian droplets were always observed to fail to reach the expected destination and divide into satellite droplets, 
halting the entire system.

Our experiments data substantiated that different behaviors in the droplet movement occurred with various interactions of chemical and biological samples. These serve as the rationale for embedding an automation of fluidic protocol. This protocol requires a feedback controller whose sensing mode is independent of liquid composition. In this work, we introduce a capacitance-based indicator that tracks the droplet position, even in the domain between two adjacent electrodes. This estimator is independent of the liquid composition because the droplet position is calculated based on a dimensionless ratio of adjacent electrodes' capacitances. Fig. 2d compared the actual position and experimental droplet position estimated from the capacitances for different liquids. The actual droplet position was calculated from the top view images. The experimental droplet position was estimated using imbed protocol. As illustrated in Fig.2d, a precise tracking scheme was achieved in our work that the position estimates of multi-ion solutions almost lay along the ideal line. The positions estimated from algae suspensions are significantly deviated from the ideal line. The negative deviation behavior occurred in the first half and positive in the second half. However, the estimates are accurate in the start, the middle, and the end points, which are likely key points for feedback control. To further confirm the proposed capacitance-based droplet movement control protocol for algae screening system, the percentage of completion of different inclusions without additional calculation has been studied (the threshold value $\mathrm{R}_{\text {thresh }}$ was set to 0.75 ). As listed in Table 1, DI water, nutrients and salty water can be transported with $100 \%$ completion with feedback controllers. Especially, droplet actuation reliability is dramatically improved for liquids containing algae (e.g. <5\% completion without feedback and $98 \%$ completion with feedback for P.helgolandica). Thus, this proposed approach significantly inspires imperfect droplet hydrodynamics of multiple liquid and algae samples in the algae screening system.

\subsection{Architecture and characterization of the DMF bioreactor}

Several design iterations were required to develop a device capable of completion of the complex sequences in algae culture and screening. Firstly, establishment of the hydrodynamic profiles by our experiments enforced an introduction of a feedback controller independent of liquid composition. However, this introduction requires complex circuitry and signal processing. The cost-adding and system-complicating continued to be problematic for DMF screening applications. To address this issue, an innovative electrode-saving scheme was applied here. In this scheme, electrodes for algae culturing are connected with "finger-fringe-bridge" geometry rather than one or more interval electrodes (Fig.3a). With these geometries, algae droplets can be addressed individually without cross-talk even when a droplet walking past in the absence of spacer electrodes (Fig.3b). Besides, an "interdigitated finger bridge" formed by finger fringes of two neighbor electrodes guaranteed algae droplets transporting more smoothly from non-charged electrode to charged one. With this "bridge", droplets are more liable to spread and increase the contact area and thus increasing the successful transportation chance (Fig.3c). The interval between two usual designed electrodes is negligible, and therefore at least one or two electrodes need to be inserted into two culturing electrodes to implement isolating and connecting functions. In $6 \times 1$ culturing electrodes, at least 5 intervals were needed. In $6 \times n$ culturing electrodes, at least $5 \times n$ intervals were needed (Fig.1e). With the finger geometries of culturing electrodes, our DMF designing was an electrode-saving scheme whose driving controller holding inherent simplicity and high fault tolerance will made our DMF system a great potential screening platform.

Besides using a smart control system compensated with electrode-saving scheme, a simple approach for a highly accurate deterministic splitting of liquids was also demonstrated. In our work, the droplet division on the electrode of splitting region was performed by powering on/off horizontal adjoining electrodes and thus activating vertical adjoining electrodes and so-called "bi-directional stretching split" (Fig. 3d). Under equilibrium conditions, the droplet maintains of equal Laplace pressures at the whole oil-water interface and can be described as a function of horizontal and vertical radii of curvatures of meniscus and interfacial tension $\gamma_{\mathrm{ci}}: p=\gamma_{\mathrm{ci}}\left(\frac{1}{R_{n}}+\frac{1}{R_{v}}\right)$ (Banerjee et al., 2012). When redistributing of volume from the splitting region to the horizontal adjoining voltage-activated regions, extra Laplace pressure will be accumulated across the highly deformed aqueous-oil interface. If the voltage at the horizontal splitting electrode is switched off abruptly, the momentum transfer (interfacial energy to internal energy) inside droplet cause an initial transient impulse, 
which makes droplet deformation along its horizontal axis toward the center. With this horizontal confining force, we observed that more deterministic fluid vertical divisions were achieved from the splitting region to the vertical adjacently electro-wetted segments. The deviation data results of DI water from 5 independent bi-direction or uni-direction splitting were shown in Fig.3e. The average volumetric errors of DI water, and salty waters with low to high salinity were all lower than $1.5 \%$ for "bi-directional stretching split" and $3 \% \sim 6 \%$ for common stretching split. Percent of abnormal droplet dividing (volume deviation [?] $\pm 20 \%$ ) in total dividing was $0 \%$ for "bi-directional stretching split" and $10 \%$ for common stretching splitting. To further verify the accuracy of concentration gradient generation, serial dilution experiments are performed. Figure $3 \mathrm{f}$ demonstrates the DI water droplets (supplemented with fluoresce dye Rh-123) generated for dilution ratios of $0,1 / 16,1 / 8,1 / 4,1 / 2$ and 1 . The intensities of Rh-123 at six droplets were measured, and compared with the theoretical data. There was a good coherence between the theoretical and experimental data with a correlation coefficient of 0.996. Since there are no significant difference between the splitting volumetric error of DI water and that of seawaters, dilution performance will be also valid for salt waters with wide range of salinity on our DMF chip. Thus, stressors triggering lipid analysis of algae can be performed in a simple and accurate way.

\subsection{DMF droplet-based interspecies lipid screening in microalgae}

Before the verification of the capability of DMF micro-bioreactor in lipid screening, their biocompatibility was initially evaluated by microalgal motility measurements and growth experiment. With the DMF microbioreactors, P.helgolandica var. tsingtaoensis could be nondestructively actuated and successfully cultured on-chip. Cell motility and concentrations for these cultures were determined using optical microscopy, with a detailed description given in Fig. S5 and supporting information.

To demonstrate the streamlined analysis functionality of the developed DMF platform, growth and oil accumulation analyses of four costal species of D. zhanjiangenis , Chlorella sp., P.helgolandicaand P.subcordiformis were performed on-chip. For further validation, off-chip experiments were also performed, in which the growth data were obtained using 96-well plate and hemocytomete and the oil production was analyzed by total lipid extraction. As illustrated in Fig.4a and 4b, both on-chip and off-chip population measurements showed similar growth resulting for each of these four algal species. On-chip growth rates are $0.249 \pm 0.007 \mathrm{~d}^{-1}, 0.313 \pm 0.004 \mathrm{~d}^{-1}, 0.248 \pm 0.03 \mathrm{~d}^{-1}$ and $0.251 \pm 0.003 \mathrm{~d}^{-1}$ for D. zhanjiangenis ,Chlorella sp., P.helgolandica and P.subcordiformi, respectively. From these growth data, Chlorella sp. was more suitable for large scale culture to manufacture biodiesel among the four strains for feature of rapid growth. Same results were also achieved by the off-chip experiments.

After 5-days culturing, intracellular lipid bodies were stained with BODIPY and imaged for oil content analyzing. Intercellular TAGs were visualized as light green fluorescence which was spectrally separated from the red algal auto-fluorescence and also varied significantly in intensity between the four species (Fig.4c). We calculated the lipid content using Image $\mathrm{J}$ software which were represented as average fluorescence intensities per unit cell area and illustrated in Fig.4d. As illustrated, interspecies lipid contents were significantly recognizable, although algae were encapsulated in DMF micro-droplets. It interpreted that oil-rich microalgae can be generally discriminated using fluorescence-activated cell sorting (FACS) on DMF (Lee et al., 2013). As expected, the same conclusion was gained from off-chip total lipid extraction data, where Chlorella sp. exhibited higher lipid content than other three and can be the selection for biodiesel production (Fig.4e).

\subsection{Stressor triggered lipid accumulation on-chip}

As we know that algae derived lipid content would be greatly inspired by proper stimuli supply (Yin et al., 2020). Determination of a specific stimuli and proper culturing condition for a certain species are costly and labor-intensive process. The DMF diluter is well suitable for the rapid and mass creating the culturing conditions responsible for lipid production. Since stressor triggered lipid accumulation in algea has not been explored in DMF droplet-based bioreactors, the following will focus on feasibility study of the DMF system.

Selected strain with high lipid productivity (oil-rich/high growth rate) of Chlorella. $s p$. was encapsulated in a series of droplets with nitrogen-depleted gradients and phosphorus-depleted gradients for 5 days and 
then the BODIPY fluorescence responses were observed. DMF-based in situ profiling of the stressor triggered growth and lipid contents within droplets were shown in Fig.5. As shown in enlarged bright-field and fluorescence images, $\mathrm{N}\left(\mathrm{NaNO}_{3}\right)$ and $\mathrm{P}\left(\mathrm{NaH}_{2} \mathrm{PO}_{4}\right)$ fertilizers have great influence on the biomass and lipid content of Chlorella sp.(Fig.5a-d). It was found that the increase of nitrogen and phosphate concentrations would promote the growth of cells to produce more biomass in droplets. However, accumulated lipid bodies, exhibited highly increasing fluorescence signals among cells with the decreasing of nutrition concentration. The lowest cell density, but the clear/largest cytosolic lipid bodies are seen in zero concentration of nutrition among all stressed cultures.

The resulting growths in all droplet-based cultures are shown in Fig.5e and 5f (blue line). Increasing of nutrition concentration would promote the growth of cells while inhibit the accumulation of oil. The most significant effect on the growth rate of Chlorella sp. was obtained when the concentrations of $\mathrm{NaNO}_{3}$ were between $2.2 \times 10^{-3}$ mol. $1^{-1} \sim 8.8 \times 10^{-3} \mathrm{~mol} . \mathrm{l}^{-1}$ (Fig. 5e, blue line) or when the concentration of $\mathrm{NaH}_{2} \mathrm{PO}_{4}$ were $0.9 \times 10^{-4}$ mol.. ${ }^{-1} \sim 3.6 \times 10^{-4}$ mol. $l^{-1}$ (Fig. 5f, blue line). Applying excessive amount of $\mathrm{NaNO}_{3}$ will not promote cell growth significantly. The cell growth will be slightly suppressed by extra $\mathrm{NaH}_{2} \mathrm{PO}_{4}$. Fluorescence intensities for representative cells in both cultures are shown in Fig. 5e and $5 \mathrm{f}$ (red column). As for oil accumulation, the nitrate-depleted culture $\left(\mathrm{NO}_{3}{ }^{-}<8.8 \times 10^{-3} \mathrm{~mol} \mathrm{l}^{-1}\right)$ has an average green fluorescence intensity of $104^{\sim} 165$ unit, while the nitrate-replete culturing $\left(\mathrm{NO}_{3}^{-}>8.8 \times 10^{-3} \mathrm{~mol} \mathrm{l}^{-1}\right)$ has an average intensity of $70^{\sim} 77$ unit (Fig. 5e, red column). At the normal P concentration of $3.6 \times 10^{-5} \mathrm{~mol} \mathrm{l}^{-1}$, the average BODIPY fluorescence intensity of Chlorella sp. was 79 unit. It is equivalent to only $45 \%$ of the P deprivation cultured cells (Fig. 5f, red column). Higher cellular lipid contents, associated with higher growth data, have addressed the optimal fertilizer supply for maximum lipid productivity, which were $2.2 \times 10^{-3} \mathrm{moll}^{-1} 4.4 \times 10^{-3} \mathrm{~mol} \mathrm{l}^{-1}$ of $\mathrm{NaNO}_{3}$ or $0.9 \times 10^{-4}$ mol l-1 $3.6 \times 10^{-4}$ of $\mathrm{NaH}_{2} \mathrm{PO}_{4}$. Together, these data suggest that lipid productivity can be stimulated in DMF bioreactors for microalgae, making screening using this technology readily attainable.

\section{Conclusions}

We demonstrated a DMF droplet-based microalgae bioreactor suitable for screening of competent species and proper fertilizer supply. A unique adaptation of capacitance-based position estimator associated with electrode-saving architecture was used, which allows for inherent simplicity and high-fidelity performance of any type of droplet. A deterministic splitting of liquids to generate nutrient gradient was also demonstrated. Using this system, microalgae were successfully cultured and stressed. In all cases, we confirmed that the results in DMF droplets were comparable to that of off-chip culturing cells, which exhibit an unparalleled potential in developing an integrated platform for fully-automatic, high-throughput microalgal lipid screening.

SUPPORTING INFORMATION Additional supporting information may be found online in the Supporting Information section.

\section{ACKNOWLEDGMENTS}

This work was supported by National Natural Science Foundation of China (No. 41476085, No. 81471807), Scientific Research Project of Liaoning Education Department (LJQ2015005) and Dalian Science and Technology Innovation Fund (2019J12SN55).

CONFLICT OF INTERESTS The authors declare that there are no conflict of interests.

\section{REFERENCE}

Banerjee, A., Liu, Y., Heikenfeld, J., Papautsky, I. (2012). Deterministic splitting of fluid volumes in electrowetting microfluidics. Lab on a Chip , 12, 5138-5141.doi:10.1039/C2LC40723J

Bligh, E.G., Dyer, W.J., Can, J. (1959). A rapid method of total lipid extraction and purification. Canada Journal of Biochemistry and Physiology, 37, 911-917. doi:10.1139/o59-099 
Bogojevic, D., Chamberlain,M.D., Barbulovic-Nad, I., Wheeler, A.R. (2012). A digital microfluidic method for multiplexed cell-based apoptosis assays. Lab on a Chip , 12, 627-634. doi: 10.1039/C2LC20893H

Chang,Y.H., Lee, G.B., Huang, F.C., Chen, Y.Y., Lin, J.L. (2006). Integrated polymerase chain reaction chips utilizing digital microfluidics. Biomedical Microdevices , 8, 215-225. doi:10.1007/s10544-006-8171-y

Chen, C.Y., Chang, H.Y. (2016). Lipid production of microalgachlorella sorokiniana CY1 is improved by light source arrangement, bioreactor operation mode and deep-Sea water supplements.Biotechnology Journal , 11(3), 356-362. doi:10.1002/biot.201500288

Choi, K., Ng A.H., Fobel, R., Wheeler, A.R. (2012). Digital microfluidics. Annual Review of Analytical Chemistry , 5, 413-440. doi:10.1146/annurev-anchem-062011-143028

Dalmazzo, D., Santagata, E., Daniel, N., Zanetti, M. (2010). Rheological characterization of sludge coming from a wastewater treatment plant.American journal of environmental sciences , 6 (4), 329337.doi:10.3844/ajessp.2010.329.337

Fan S. K., Yang H., Hsu W. (2011) Droplet-on-a-wristband: Chip-to-chip digital microfluidic interfaces between replaceable and flexible electrowetting modules. Lab On a Chip , 2, 343-347.doi:10.1039/C0LC00178C

Gao, J., Liu, X., Chen, T., Mak, P.I., Du, Y., Vai, M.I., Lin, B., Martins, R.P. (2013). An intelligent digital microfluidic system with fuzzy-enhanced feedback for multi-droplet manipulation. Lab on a Chip, 13, 443451.doi:10.1039/C2LC41156C

Gim, G.H., Ryu, J., Kim, M.J., Kim, P.I., Kim, S.W. (2016). Effects of carbon source and light intensity on the growth and total lipid production of three microalgae under different culture conditions. Journal of Industrial Microbiology \& Biotechnology , 43, 605-616. doi:10.1007/s10295-016-1741-y

Gocze, P.M., Freeman, D.A. (1994). Factors underlying the variability of lipid droplet fluorescence in Ma-10 Leydig tumor cells.Cytometry , 17, 151-158. doi:10.1002/cyto.990170207

Hribar, B., Southall, N.T., Vlachy, V., Dill, K.A. (2002). How ions affect the structure of water.Journal of the American Chemical Society. 124, 12302-12311.doi:10.1021/ja026014h

Han S, Zhang Q, Zhang X, Liu X, Lu L, Wei J, Li Y, Wang Y, Zheng G. (2019). A digital microfluidic diluter based microalgal motion biosensor for marine pollution monitoring. Biosensors and Bioelectronics . 143:111597.doi:10.1016/j.bios.2019.111597

Hu, Q., Sommerfeld, M., Jarvis, E., Ghirardi, M., Posewitz, M., Seibert, M., Darzins, A. (2008). Microalgal triacylglycerols as feedstocks for biofuel production: perspectives and advances. The Plant Journal.54(4), 621-639. doi:10.1111/j.1365-313X.2008.03492.x

Jin H, Zhang H, Zhou Z, Li K., Hou G., Xu Q., Chuai W., Zhang C., Han D., Hu Q. (2020) Ultrahigh-celldensity heterotrophic cultivation of the unicellular green microalga Scenedesmus acuminatus and application of the cells to photoautotrophic culture enhance biomass and lipid production. Biotechnology and Bioengineering ,117, 96-108. doi:10.1002/bit.27190

Josep P L (2014). Microfluidic platforms: a mainstream technology for the preparation of crystals. Chemical Society Reviews, 43, 7, 2253-2271.doi:10.1039/C3CS60372E

Khandelwal, A., Chhabra, M., Yadav, P. (2020). Performance evaluation of algae assisted microbial fuel cell under outdoor conditions.Bioresource Technology , 310, 123418. doi:10.1016/j.biortech.2020.123418

Lee, D.H., Bae, C.Y., Han, J.I., Park, J.K. (2013).In situ analysis of heterogeneity in the lipid content of Single Green Microalgae in alginate hydrogel microcapsules. Analytical Chemistry , 85, 87498756.doi:10.1021/ac401836j

Malic, L., Veres,T., Tabrizian, M. (2009). Biochip functionalization using electrowetting-on- dielectric digital microfluidics for surface plasmon resonance imaging detection of DNA hybridization.Biosensors and 
Bioelectronics, 24(7), 2218-2224. doi:10.1016/j.bios.2008.11.031

Marcus, Y.J. (1994). Viscosity B-coefficients, structural entropies and heat capacities, and the effects of ions on the structure of water.Journal of Solution Chemistry, 23, 831-848. doi:10.1007/BF00972677

Marcus, Y.J. 2015. The Effect of Complex Anions on the Structure of Water. Journal of Solution Chemistry, 44, 2258-2265.doi:10.1007/s10953-015-0402-5

Mueller, S.P., Llewellin, E.W., Mader, H.M. (2010). The rheology of suspensions of solid particles. Proceedings of the Royal Society A , 466, 1201-1228.doi:10.1098/rspa.2009.0445

Rackus DG, Dryden MD, Lamanna J, Zaragoza A, Lam B, Kelley SO, Wheeler AR (2015). A digital microfluidic device with integrated nanostructured microelectrodes for electrochemical immunoassays. Lab on a Chip , 15(18), 3776-84. doi:10.1039/c5lc00660k.

Richter, L.V., Mansfeldt, C.B., Kuan, M.M., Cesare, A.E., Menefee, S.T., Richardson, R.E., Ahner, B.A. (2018). Altered Microbiome Leads to Significant Phenotypic and Transcriptomic Differences in a LipidAccumulating. Environmental Science and Technology , 52, 6854-6863.doi:10.1021/acs.est.7b06581

Schell, W.A., Benton, J.L., Smith, P.B. (2012). Evaluation of a digital microfluidic real-time PCR platform to detect DNA of Candida albicans in blood. European Journal of Clinical Microbiology \& Infectious Diseases . 31, 2237-2245. doi:10.1007/s10096-012-1561-6

Shih, S.C.C., Fobel, R., Kumar, P., Wheeler, A.R. (2011). A feedback control system for high-fidelity digital microfluidics. Lab on a Chip , 11(3), 535-540.doi:10.1039/C0LC00223B

Shin, Y.J., Lee, J.B. (2010). Machine vision for digital microfluidics. The Review of Society Instruments, 81, 014302. doi:10.1063/1.3274673

Shukla, V., Hussin, F.A., Hamid, N.H., Zain Ali, N.B. (2017). Advances in testing techniques for digital microfluidic biochips. Sensors (Basel) ,17(8), 1719-1726.doi:10.3390/s17081719

Vergauwe,N., Witters,D., Ceyssens, F., Vermeir, S., Verbruggen,B., Puers, R., Lammertyn, J.J. (2011). A versatile electrowetting-based digital microfluidic platform for quantitative homogeneous and heterogeneous bioassays. Journal of Micromechanics and Microengineering, 21, 054026.doi:10.1088/0960-1317/21/5/054026

Wang, Y.B., Huang, J.H., Lee, M.S., Huang, C.Y., Huang, C.S., Yamashita, I., Tu, Y.Y., Hsu, W., (2017). An EWOD-based micro diluter with high flexibility on dilution ratio. Microsystem Technology. 23, 36453651. doi:10.1007/s00542-016-3146-2

Yin, Z.H., Zhu, L.D., Li, S.X., Hu, T.Y., Chu. R.Y., Mo, F., Hu, D., Liu, C.C., Li, B. (2020). A comprehensive review on cultivation and harvesting of microalgae for biodiesel production: Environmental pollution control and future directions. Bioresource Technology , 301, 122804. doi:10.1016/j.biortech.2020.122804

Zhang, Q., Zhang, X.C., Zhang, X.L., Jiang, L., Yin, J.M., Zhang, P., Han, S., Wang, Y.H., Zheng G.X. (2019). A feedback-controlling digital microfluidic fluorimetric sensor device for simple and rapid detection of mercury (II) in costal seawater. Marine Pollution. Bulletin , 144, 20-27.doi:10.1016/j.marpolbul.2019.04.063

\section{TABLE}

Table 1 Transport of multi-liquid with and without feedback control

+ No sign represents the percent of successful transportations on actuation electrodes and $*$ represents that on culturing electrodes

\section{FIGURE LEGENDS}


Fig. 1. Proposed control-engaged DMF screening technology. Overview schematic of the DMF screening system that (a) the DMF chip demonstration is patterned a 24-electrodes array which is connected to (b) the operation unit with position feedback for actuating and mounted onto (c) the detection element for detecting. (d) DMF chip with a 6x3culturing electrodes array (e) Schematic of the DMF chip with a $6 \mathrm{x} n$ culturing electrodes array.

Fig.2. Droplet movement for DMF actuation of droplets of complex liquids containing (a) Nutrients (b) Salty water and (c) Algae, without feedback. (d) Actual and estimated droplet position plot from experiments.

Fig.3. Design of DMF screening system.(a) The configuration of culturing electrodes. It has one or two finger fringes to form "Interdigitated-finger Bridge" for connecting with each other. The "Bridge" functions of (b) isolating and (c) transporting droplets. (d) Droplet bi-directional stretching split way used in this study compared with common split way. The power goes through the electrode displayed by yellowsign. (e) Deviation of target volume of DI water on one side during random 5 merge-split cycles.(f) Dilution performance validation. As the fluorescence intensity of Rh-123 was proportional to the concentration, the intensities of Rh-123 at six droplets generated by DMF diluter were imaged by a fluorescence microscope.

Fig.4. Interspecies comparison of lipid productivity in microalgae. (a) On-chip growth analysis of four microalgal species. The number of the algae were counted. The growth rates were calculated. (b) Off-chip growth analysis of four microalgal species.(c) On-chip fluorescence images of the four species respectively, stained with BODIPY. The autofluorescence (red) and oil fluorescence (BODIPY, green) were shown in upper-row. The oil fluorescence were shown in bottom row. (d) Algal cells were cultured in droplet (on chip) for 5 days. The cells were then stained with BODIPY and average fluorescence intensities were analyzed for measurement of oil accumulation. (e) Off-chip measurements of oil accumulation for 5 days of culture.

Fig.5. In situ profiling of the stressor triggered growth and lipid contents within DMF droplets. (a) Enlarged bright-field images of algae cells in droplets and (b) fluorescence images of Cytosolic lipid bodies in representative cells cultured for 5 days in nitrate-deplete mediums. (c) Enlarged bright-field images of algae cells in droplets and (d) fluorescence images of Cytosolic lipid bodies in representative cells cultured for 5 days in phosphate-depletemediums.(e)The growth (blue line) and oil accumulation (red column) of Chlorella $s p$.cultured in nitrate-depletemediums were analyzed. (f) The growth (blue line) and oil accumulation (red column) of Chlorella sp. cultured inphosphate-deplete mediums were analyzed.

\section{Hosted file}

Table 1.docx available at https://authorea.com/users/347082/articles/472907-an-integrateddigital-microfluidic-bioreactor-for-fully-automatic-screening-of-microalgal-growth-andstress-induced-lipid-accumulation 

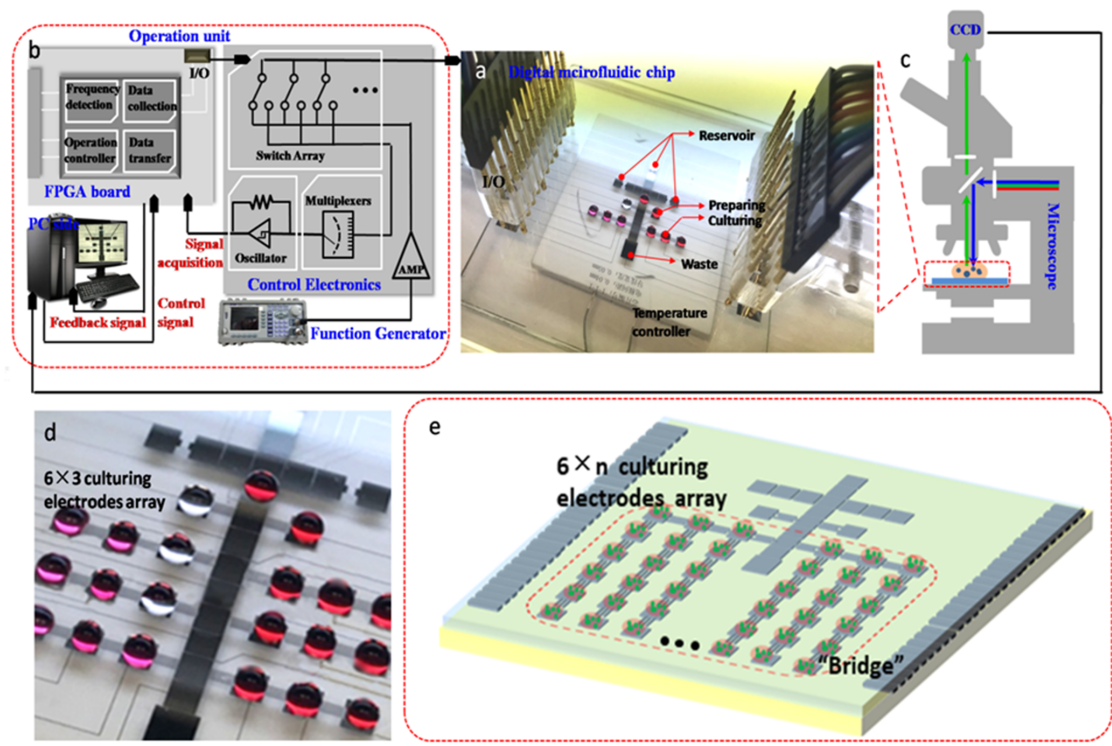

\section{Hosted file}

Figure 2.eps available at https://authorea.com/users/347082/articles/472907-an-integrateddigital-microfluidic-bioreactor-for-fully-automatic-screening-of-microalgal-growth-andstress-induced-lipid-accumulation

\section{Hosted file}

Figure 3.eps available at https://authorea.com/users/347082/articles/472907-an-integrateddigital-microfluidic-bioreactor-for-fully-automatic-screening-of-microalgal-growth-andstress-induced-lipid-accumulation

\section{Hosted file}

Figure 4.eps available at https://authorea.com/users/347082/articles/472907-an-integrateddigital-microfluidic-bioreactor-for-fully-automatic-screening-of-microalgal-growth-andstress-induced-lipid-accumulation

\section{Hosted file}

Figure 5.eps available at https://authorea.com/users/347082/articles/472907-an-integrateddigital-microfluidic-bioreactor-for-fully-automatic-screening-of-microalgal-growth-andstress-induced-lipid-accumulation 